\title{
Effect of a dc magnetic field on the anomalous dispersion of localized Josephson plasma modes in layered superconductors
}

\author{
T. Rokhmanova ${ }^{1,2}$, S.S. Apostolov ${ }^{1,2}$, N. Kvitka ${ }^{2}$, and V.A. Yampol'skii ${ }^{1,2}$ \\ ${ }^{1}$ A.Ya. Usikov Institute for Radiophysics and Electronics NASU, Kharkiv 61085, Ukraine \\ ${ }^{2}$ V.N. Karazin Kharkiv National University, Kharkiv 61077, Ukraine \\ E-mail: Rokhmanova@ieee.org
}

Received December 27, 2017, published online April 25, 2018

\begin{abstract}
We study theoretically the propagation of Josephson plasma waves (JPWs) localized on a slab of layered superconductor in the presence of an external dc magnetic field. The slab is sandwiched between two dielectric half-spaces and the wave modes propagate across the layers. We derive analytic expressions for the dispersion relations of the localized JPWs and present the numerical simulation for the effect of the external dc magnetic field on the dispersion. The anomalous dispersion of localized JPWs is predicted for a wide range of frequencies, wave vectors, and dc fields. Also, we discuss the possibility of the internal reflection of the localized modes in the inhomogeneous dc magnetic field. This phenomenon can find application in the terahertz electronics for the control of the localized mode propagation.
\end{abstract}

PACS: 74.72.-h Cuprate superconductors;

73.20.Mf Collective excitations (including excitons, polarons, plasmons and other charge-density excitations);

52.35.Mw Nonlinear phenomena: waves, wave propagation, and other interactions.

Keywords: layered superconductors, localized Josephson plasma waves, dc magnetic field, anomalous dispersion.

\section{Introduction}

Layered superconductors are periodic materials, where thin superconducting layers are separated by thicker insulating ones and are electrodynamically related to each other by means of the intrinsic Josephson effect (see Fig. 1). The experimental confirmation of such an electrodynamic model for layered superconductors can be found in Refs. 1, 2 . High-temperature superconductors based on $\mathrm{Bi}$, $\mathrm{La}$, or $\mathrm{Y}$ with $\mathrm{CuO}_{2}$ superconducting layers belong to this family of materials. The essential property of layered superconductors is the considerable anisotropy of their current-carrying capability. The current along the layers is of the same nature as in the bulk superconductors and is much stronger than the current across the layers caused by the Josephson effect. Thus, the so-called Josephson plasma is formed in layered superconductors. This strongly anisotropic Josephson solidstate plasma supports the propagation of the specific excitations in layered superconductors, the Josephson plasma waves (JPWs). These waves belong to the terahertz frequen- cy range, which makes layered superconductors interesting for terahertz electronics (see, e.g., Ref. 3). In turn, the terahertz technologies are promising for various applications, including medical diagnostics and security control (see, e.g., Ref. 4). Furthermore, the study of the interaction of strong terahertz pulses with layered superconductors (see experi-

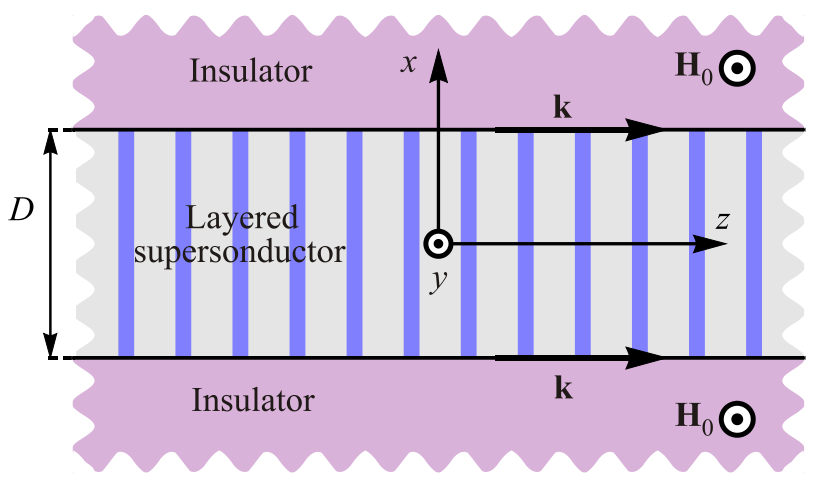

Fig. 1. Sketch of the setup. Here $D$ is the thickness of the sample, $\mathbf{H}_{0}$ is the external dc magnetic field, $\mathbf{k}$ is the wave vector. 
mental works Refs. 5,6,7) may reveal new possibilities for high-temperature superconductive state control.

In the Josephson plasma, various interesting electrodynamic phenomena can be observed, both common and uncommon to the other types of plasmas. As was theoretically demonstrated in Refs. 8, 9, the surface JPWs can propagate along the interface between the layered superconductor and external dielectric, similarly to surface plasmonpolaritons in usual plasmas. The excitation of these waves leads to various resonant phenomena $[9,11,12]$ similar to the Wood anomalies well known in optics (see Refs. 13-15). However, contrary to usual plasmas, the surface JPWs can propagate with frequencies not only below the plasma frequency but also above it [9]. As was shown in Ref. 16, the phenomena similar to the Anderson localization and the formation of a transparency window for $\mathrm{THz}$ waves can be observed in layered superconductors with randomlyfluctuating value of the maximum Josephson current.

As was described in Ref. 17, when the layers are perpendicular to the slab boundaries and to the direction of JPWs propagation, the anomalous dispersion of the localized waves can be observed in layered superconductors in a certain range of frequencies and wave numbers. It is caused, in particular, by the different signs of the longitudinal and transversal components of the effective permittivity tensor in layered superconductors. A system containing material with anomalous dispersion can have negative refractive index. Refs. 9, 10 present evidence of the negative refractive index for the surface JPWs in layered superconductors above the plasma frequency. Although the negative index materials were mentioned earlier in the literature (see Ref. 18), they started to attract great attention after 2000, when a theoretical prediction of a perfect lens creation using such materials was presented in Ref. 19. Since then a great amount of works have been carried out resulting rapid development of this field. Some reviews of the recent advances can be found in Refs. 20, 21.

The possibility of the anomalous dispersion manipulation is promising for various applications. dc magnetic field is one of the tools that can flexibly change the electromagnetic properties of layered superconductors. In Refs. 22, 23, the effect of the weak external dc magnetic field on the Terahertz waves transmission, reflection, and polarization transformation in layered superconductors was studied theoretically. It turned out that even relatively weak magnetic field can significantly change the conditions for the waves propagation. Therefore, the external dc magnetic field turns up to be an interesting tool to control the localized JPWs in layered superconductors.

In the present work, we study theoretically how the relatively weak external dc magnetic field affects the dispersion properties of the localized JPWs. The paper is organized as follows. In the second section of the paper, the studied model is presented. There are presented the geometry of the problem and the main equations for romagnetic fields. The third section is devoted to the derivation of the dispersion relations for the localized modes in the WKB approximation and in the exact form in terms of the special Legendre functions. The fourth section contains analysis of the obtained relations, where we consider the effect of the external dc magnetic field on the dispersion curves. In the fifth section, we discuss the possibility of the internal reflection of the localized modes in the inhomogeneous dc magnetic field. This phenomenon can find application in the terahertz electronics for the control of the localized mode propagation. The obtained results are summarized in the conclusions.

\section{Model}

We study the linear localized JPWs propagating in a slab of layered superconductor sandwiched between two dielectric half-spaces (see Fig. 1). The layers are perpendicular to the boundaries of the slab. The coordinate system is chosen in such a way that the $z$-axis is directed across the superconducting layers, i.e., along the crystallographic $c$-axis, and parallel to the boundaries of the slab. The $x$ - and $y$-axes are directed along the superconducting layers, i.e., along the $a b$ plane. The $x$-axis is perpendicular to the slab boundaries, while the $y$-axis is parallel to them. The slab of the thickness $D$ is located at $|x|<D / 2$, where the upper and lower dielectric half-spaces with the permittivity $\varepsilon_{d}$ occupy the regions $x>D / 2$ and $x<-D / 2$, respectively. Thus, the plane $x=0$ is in the middle of the slab and divides the system into two symmetrical parts. The external dc magnetic field $\mathbf{H}_{0}$ is directed along the $y$-axis and is uniformly distributed outside the slab of layered superconductor.

We consider the localized JPWs of the following polarization:

$$
\begin{gathered}
\mathbf{H}(x, y, z, t)=\left\{0, H_{y}(x), 0\right\} \exp \left(i k_{z} z-i \omega t\right), \\
\mathbf{E}(x, y, z, t)=\left\{E_{x}(x), 0, E_{z}(x)\right\} \exp \left(i k_{z} z-i \omega t\right),
\end{gathered}
$$

where $\omega$ is the frequency of the localized mode that propagates along the $z$-axis, i.e., $k_{y}=0$.

\subsection{Electromagnetic field in the dielectric}

The JPWs are localized near the slab and evanesce far from the slab in the dielectric half-spaces. Thus, from Maxwell equation, we can obtain expressions for the components $H_{y}$ and $E_{z}$ of the electromagnetic wave,

$$
\begin{gathered}
H_{y}^{ \pm}(x)=H^{ \pm} \exp \left[\mp k_{d}(x \mp D / 2)\right], \\
E_{z}^{ \pm}(x)=\mp \frac{i c k_{d}}{\varepsilon_{d} \omega} H^{ \pm} \exp \left[\mp k_{d}(x \mp D / 2)\right],
\end{gathered}
$$

where superscripts “+ " and “ - " mean the upper $(x>D / 2)$ and lower $(x<-D / 2)$ half-spaces, respectively, $H^{ \pm}$is the amplitude of the magnetic field. The decrement $k_{d}$, 


$$
k_{d}^{2}=k_{z}^{2}-\varepsilon_{d} \omega^{2} / c^{2}>0,
$$

determines how quickly the localized mode evanesces from the slab.

\subsection{Main equations for the layered superconductor}

We assume that the period $d$ of the layered structure of the superconductor is much smaller than the wavelength across the layers, $k_{z} d \ll 1$. Therefore, we can present the electrodynamic equations for layered superconductors in the continual limit. We use wave equation for the vector potential A, which is common for macroscopic electrodynamics (some more explanations one can find in Ref. 24),

$$
\operatorname{graddiv} \mathbf{A}-\Delta \mathbf{A}=-\frac{\varepsilon_{s}}{c^{2}} \frac{\partial^{2} \mathbf{A}}{\partial t^{2}}+\frac{4 \pi}{c} \mathbf{J},
$$

where $\varepsilon_{S}$ is dielectric constant of the insulating layers in the superconductor. The components $J_{x}$ and $J_{y}$ of the current density $\mathbf{J}$ along the layers are described within the London model,

$$
J_{x}=-\frac{c}{4 \pi \lambda_{a b}^{2}} A_{x}, \quad J_{y}=-\frac{c}{4 \pi \lambda_{a b}^{2}} A_{y},
$$

where $\lambda_{a b}$ is the London penetration depth in the $c$-axis direction, while the current density $J_{z}$ across the layers is described by the Josephson relation,

$$
J_{Z}=J_{C} \sin \varphi \text {. }
$$

Here $J_{c}$ is the maximum Josephson current density and $\varphi$ is the gauge invariant interlayer phase difference [25] between neighboring layers.

The vector potential $\mathbf{A}$ is related to the electric $\mathbf{E}$ and magnetic $\mathbf{H}$ fields by the standard equations,

$$
\mathbf{H}=\operatorname{rot} \mathbf{A}, \quad \mathbf{E}=-\frac{1}{c} \frac{\partial \mathbf{A}}{\partial t},
$$

and the scalar potential is supposed to be equal to zero.

The z-component of the electric field produces the breakdown of electro-neutrality of superconducting layers, which causes additional, so-called capacitive, interlayer coupling. According to Ref. 26, the capacitive coupling is substantial only for longitudinal JPWs with frequencies close to the Josephson plasma frequency $\omega_{J}$. In this paper, we can neglect the capacitive coupling due to the smallness of the capacitive coupling parameter, $\alpha=R_{D}^{2} \varepsilon_{s} / s d \ll 1$, where $R_{D}$ is Debye length for a charge in the superconductor, $s$ is the thickness of the superconducting layers $(s \sim 1 \mathrm{~nm})$. Then the following relation between the gauge invariant interlayer phase difference $\varphi$ and the $z$-component of vector potential is valid:

$$
A_{z}=-\frac{\Phi_{0}}{2 \pi d} \varphi
$$

where $\Phi_{0}=\pi c \hbar / e$ is the magnetic flux quantum, $e$ is the elementary charge, and $c$ is the speed of light.
It should be noted that from the wave Eq. (4), using Eqs. (5) and (6), one can obtain well-known coupled sineGordon equation which is widely used in electrodynamic description of layered superconductors (see, e.g. Ref. 25). For sufficiently small frequencies, $\omega \ll \gamma \omega_{J}$, this equation takes the following form,

$$
\left(1-\lambda_{a b}^{2} \frac{\partial^{2}}{\partial z^{2}}\right)\left[\frac{1}{\omega_{J}^{2}} \frac{\partial^{2} \varphi}{\partial t^{2}}+\sin \varphi\right]-\lambda_{c}^{2} \frac{\partial^{2} \varphi}{\partial x^{2}}=0,
$$

where $\lambda_{c}=c /\left(\omega_{J} \sqrt{\varepsilon_{s}}\right)$ is the London penetration depth along the layers, $\omega_{J}=\sqrt{8 \pi e d J_{c} / \hbar \varepsilon_{s}}$ is the Josephson plasma frequency, $\gamma=\lambda_{c} / \lambda_{a b}$ is the anisotropy parameter.

\section{3. dc magnetic field in the layered superconductor}

Here we describe how the external dc magnetic field penetrates inside the slab of the layered superconductor. In this paper, we study the case of relatively small magnetic fields, $H_{0}<\mathcal{H}_{0}=\Phi_{0} / \pi d \lambda_{c}$, when the Josephson vortices do not penetrate into the superconductor. For estimations, the value of $\mathcal{H}_{0}$ for $\mathrm{Bi}_{2} \mathrm{Sr}_{2} \mathrm{CaCu}_{2} \mathrm{O}_{8+\delta}$ (with $d=1.5 \cdot 10^{-7} \mathrm{~cm}$ and $\lambda_{c}=4 \cdot 10^{-3} \mathrm{~cm}$ ) is about 100 Oe. In addition, we suppose that the superconducting slab is sufficiently thick,

$$
\exp \left(D / \lambda_{c}\right) \gg 1
$$

In this case, the dc magnetic field penetrates into the layered superconductor over small distances in the form of the tails of two fictitious vortices, each near the corresponding interfaces.

Each vortex tail can be described by the well-known solution [27] of the sine-Gordon equation (9),

$$
\varphi_{ \pm}(\xi)=\mp 4 \arctan \left[\exp \left(\xi_{0} \pm \xi\right)\right],
$$

where subscripts " + ” and " - " mean the upper $(x=D / 2)$ and lower $(x=-D / 2)$ interfaces, respectively, near which the vortex tails exist, and $\xi=x / \lambda_{C}$ is normalized coordinate. The constant $\xi_{0}$ corresponds to the center of the fictitious vortex and is defined by the normalized magnitude $h_{0}$ of the external dc magnetic field and the normalized half-thickness $\delta$ of the slab,

$$
\xi_{0}=\delta+\operatorname{arccosh}\left(h_{0}^{-1}\right), \quad \delta=D / 2 \lambda_{c}, \quad h_{0}=H_{0} / \mathcal{H}_{0} .
$$

\subsection{Electromagnetic field in the layered superconductor}

In order to describe the wave propagation in the slab of layered superconductor, we present $\varphi$ as a sum of static solutions $\varphi_{ \pm}(\xi)$, caused by the dc magnetic field, Eq. (11), and a small additive $\varphi_{\operatorname{lm}}(\xi, z, t)$ induced by the localized mode,

$$
\varphi(\xi, z, t)=\varphi_{\operatorname{lm}}(\xi, z, t)+\varphi_{+}(\xi)+\varphi_{-}(\xi)
$$

We seek $\varphi_{\operatorname{lm}}(\xi, z, t)$ in the form of the wave propagating along the $z$-axis, 


$$
\left.\varphi_{\operatorname{lm}}(\xi, z, t)=a(\xi) \exp \left[i k_{z} z-i \omega t\right)\right]
$$

Excluding the $x$ - and $y$-components of the vector potential from the wave equation (4) and linearizing $\sin \varphi \approx \varphi$, we derive the equation for the amplitude $a(\xi)$,

$$
-\frac{a^{\prime \prime}(\xi)}{\kappa_{s}^{2}}+[u(\xi)-1] a(\xi)=0,
$$

where the prime denotes derivative with respect to $\xi$,

$$
u(\xi)=\left(1-\Omega^{2}\right)^{-1}\left[\frac{2}{\cosh ^{2}\left(\xi_{0}-\xi\right)}+\frac{2}{\cosh ^{2}\left(\xi_{0}+\xi\right)}\right],
$$

and $\Omega=\omega / \omega_{J}$ is the normalized frequency. Parameter $\kappa_{s}$ represents the normalized $x$-projection of the wave vector in the absence of the dc magnetic field,

$$
\kappa_{s}^{2}=\left(\Omega^{2}-1\right)\left(1+\frac{\kappa_{z}^{2}}{1-\Omega^{2} / \gamma^{2}}\right),
$$

and $\kappa_{z}=k_{z} \lambda_{a b}$ is the normalized $z$-projection of the wave vector.

Using Eq. (7), we can express the components $H_{y}^{s}$ and $E_{z}^{S}$ of the electromagnetic field in the slab via the function $a(\xi)$,

$$
\begin{gathered}
H_{y}^{s}(\xi)=\mathcal{H}_{0} \frac{a^{\prime}(\xi)}{1+\kappa_{z}^{2} /\left(1-\Omega^{2} / \gamma^{2}\right)}, \\
E_{z}^{s}(\xi)=-\mathcal{H}_{0} \frac{i \Omega}{\sqrt{\varepsilon}} a(\xi) .
\end{gathered}
$$

In the next section we present the analytic solution of Eq. (15) and derive the dispersion relations for the localized modes.

\section{Dispersion relations}

In order to derive the dispersion relation for the localized JPWs in the slab of layered superconductor, we match the tangential components of the electric and magnetic fields at the interfaces of the slab,

$$
\left.\frac{E_{z}^{ \pm}}{H_{y}^{ \pm}}\right|_{\xi= \pm \delta}=\left.\frac{E_{z}^{s}}{H_{y}^{s}}\right|_{\xi= \pm \delta} \text {. }
$$

Rewriting this equation in terms of the amplitude $a(\xi)$, see Eqs. (2) and (18), we achieve the following relations:

$$
\frac{a^{\prime}(\xi= \pm \delta)}{a(\xi= \pm \delta)}= \pm \frac{\varepsilon^{-1} \Omega^{2} \kappa_{s}^{2}}{\left(\Omega^{2}-1\right) \kappa_{d}}
$$

where $\varepsilon=\varepsilon_{s} / \varepsilon_{d}$ and $\kappa_{d}$ represents the normalized spatial decrement for the dielectric half-spaces, see Eq. (3),

$$
\kappa_{d}^{2}=\gamma^{2} \kappa_{z}^{2}-\varepsilon^{-1} \Omega^{2}>0
$$

It should be noted that the symmetry of the studied system implies the symmetry of the localized modes, symmetric and antisymmetric with respect to the magnetic field. Therefore, we can use relation (20) only for upper interface, $\xi=+\delta$, but impose additional conditions in the middle of the slab,

$$
a(0)=0 \quad \text { or } \quad a^{\prime}(0)=0
$$

for symmetric or antisymmetric mode, respectively.

Differential equation (15) with condition (20) at $\xi=+\delta$ and one of conditions (22) define the spectrum of the localized modes. In the following subsections 3.1 and 3.2 we present the asymptotic and exact solutions of Eq. (15), respectively.

\subsection{Dispersion relations within the WKB approximation}

In this subsection, we solve Eq. (15) asymptotically. We restrict our study to the relatively low frequency range, $\omega<\gamma \omega_{J}$. On the one hand, in this frequency range all the features of the anomalous dispersion affected by dc magnetic field can be observed. On the other hand, the high frequency range is hardly attained in the experiment because of destroying the superconducting state. It should be emphasized that Eq. (15) resembles the one-dimensional Schrödinger equation with 1 standing instead of the total energy and with $u(\xi)$ instead of the potential energy. Therefore, in the case

$$
\kappa_{s} \gg 1,
$$

we can solve this equation by means of the WKB (quasiclassical) approximation. In turn, inequality Eq. (23) is satisfied under following conditions:

$$
\kappa_{z}^{2} \gg 1-\Omega^{2} / \gamma^{2}, \quad|\Omega-1| \gtrsim 1
$$

\subsubsection{Frequencies higher than $\omega_{J}$}

We start our analysis from the case of relatively high frequencies, $1<\Omega<\gamma$, when the parameter $\kappa_{s}$ is positive (see Eq. (17)) and the potential energy $u(\xi)$ is negative (see Eq. (16)). In this case, the classical turning points are absent and the WKB solution of (15) can be presented as

$$
\begin{aligned}
& a(\xi)=\frac{a_{\mathrm{sym}}^{\mathrm{WKB}}}{\sqrt{b^{\prime}(\xi)}} \sin \left[\kappa_{s} b(\xi)\right], \\
& a(\xi)=\frac{a_{\mathrm{asym}}^{\mathrm{WKB}}}{\sqrt{b^{\prime}(\xi)}} \cos \left[\kappa_{s} b(\xi)\right],
\end{aligned}
$$

for the symmetric and antisymmetric with respect to magnetic field modes. Here $a_{\mathrm{asym}}^{\mathrm{WKB}}$ and $a_{\mathrm{sym}}^{\mathrm{WKB}}$ are the integration constants,

$$
b(\xi)=\int_{0}^{\xi} \sqrt{1-u\left(\xi^{\prime}\right)} d \xi^{\prime} .
$$


In order to derive the dispersion relations, we substitute solutions (25) into Eq. (20). As a result, we get the dispersion relations,

$$
\cot \left[\kappa_{s} b(\delta)\right]=\beta, \quad \tan \left[\kappa_{s} b(\delta)\right]=-\beta,
$$

for the symmetric and antisymmetric localized modes. Here

$$
\beta=\frac{\varepsilon^{-1} \Omega^{2} \kappa_{s}}{\left(\Omega^{2}-1\right) \kappa_{d}}\left[1+\frac{2 h_{0}^{2}}{\Omega^{2}-1}\right]^{-1 / 2}
$$

If the z-projection of the wave vector is sufficiently large, $\kappa_{z} \gg \varepsilon^{-1 / 2} \Omega / \gamma$, then the parameter $\beta$ is small, $\beta \sim(\varepsilon \gamma)^{-1} \ll 1$. In this case, we can simplify the dispersion relation to the form $\kappa_{s} b(\delta)=\pi(n-2) / 2$, where integer $n=3,4, \ldots$ numerates the dispersion curves from bottom to top (see section 4 and Fig. 4). The odd numbers $n=3,5, \ldots$ correspond to the symmetric modes whereas even $n=4,6, \ldots$ describe the antisymmetric ones. Note that we start the numeration from $n=3$ because the dispersion curves with numbers $n=1$ and $n=2$ are located in the lower frequency range, $\Omega<1$. The last implicit dispersion relation can be rewritten in the explicit form for $\kappa_{z}(\Omega)$,

$$
\kappa_{Z}^{2}(\Omega)=\left(1-\frac{\Omega^{2}}{\gamma^{2}}\right)\left\{\frac{[\pi(n-2) / 2]^{2}}{\left(\Omega^{2}-1\right) b^{2}(\delta)}-1\right\} .
$$

\subsubsection{Frequencies lower than $\omega_{J}$}

Now we proceed to the low frequency range, $\Omega<1$. In this case, the electromagnetic field in the layered superconductor evanesces across the slab, and the wave in the slab can be represented as two weakly coupled surface modes localized near interfaces $x=D / 2$ and $x=-D / 2$. Therefore, the spectrum of such modes nearly coincides with the spectrum of the surface modes localized on the interface between the half-infinite layered superconductor and the half-infinite dielectric. The spectrum of these surface waves was studied in Ref. 27.

In the low frequency range, the parameter $\kappa_{s}$ is negative, see Eq. (17), and the potential energy $u(\xi)$ is positive, see Eq. (16). This means that, under conditions

$$
\frac{4 h_{0}^{2} \exp (-2 \delta)}{\left(1+\sqrt{1-h_{0}^{2}}\right)^{2}}<1-\Omega^{2}<2 h_{0}^{2},
$$

there exist classical turning points, $\xi= \pm \xi_{t p}$, defined by the equation $u\left(\xi= \pm \xi_{t p}\right)=1$, or, according to Eq. (16),

$$
\cosh ^{2}\left(\xi_{0}-\xi_{t p}\right)=\frac{2}{1-\Omega^{2}} .
$$

Here we keep only one summand $\cosh ^{-2}\left(\xi_{t p}-\xi_{0}\right)$ in Eq. (16) because the other summand, $\cosh ^{-2}\left(\xi_{t p}+\xi_{0}\right)$, is exponentially small. It should be noted that, under assumptions (10) and (24), the left-hand inequality in Eq. (30) is satisfied for arbitrary $h_{0}$.

The WKB solution of Eq. (15) with classical turning point $\xi_{t p}$ can be presented in the following form:

$$
a(\xi)= \begin{cases}\frac{a^{\mathrm{WKB}}}{\sqrt{b^{\prime}(\xi)}} \cos \left[\left|\kappa_{s}\right| \bar{b}(\xi)-\pi / 4\right], & \xi_{t p}<\xi<\delta, \\ \frac{a^{\mathrm{WKB}} / 2}{\sqrt{b^{\prime}(\xi)}} \exp \left[-\left|\kappa_{s} \bar{b}(\xi)\right|\right], & 0<\xi<\xi_{t p},\end{cases}
$$

where $a^{\mathrm{WKB}}$ is the integration constant and

$$
\bar{b}(\xi)=\int_{\xi_{t p}}^{\xi} \sqrt{u\left(\xi^{\prime}\right)-1} d \xi^{\prime} .
$$

The first line in Eq. (32) corresponds to the classically allowed region, $\xi_{t p}<\xi<\delta$, while the second line describes the classically forbidden zone, $0<\xi<\xi_{t p}$. Formula (32) presents the field near upper interface $\xi=+\delta$ only. This solution is valid when $\exp \left(-2\left|\kappa_{s} \bar{b}(0)\right|\right) \ll 1$, and we can neglect the weak coupling with the field near lower interface, $\xi=-\delta$, writing “exp” instead of “ $\cosh$ ” or “ sinh ” in the classically forbidden zone.

Applying solution (32) in Eq. (20), we derive the dispersion relation

$$
\tan \left[\left|\kappa_{s}\right| \bar{b}(\delta)-\pi / 4\right]=-\beta
$$

for weakly coupled modes localized near interfaces $\xi= \pm \delta$. Here $\beta$ is defined by Eq. (28). If the $z$-projection of the wave vector is sufficiently large, $\kappa_{z} \gg \varepsilon^{-1 / 2} \Omega / \gamma$, the parameter $\beta$ is small, $\beta \ll 1$. In this case, we can simplify the dispersion relation to $\left|\kappa_{s}\right| \bar{b}(\delta)=\pi(m+1 / 4)$, where $m=0,1,2, \ldots$, and rewrite it in the explicit form for $\kappa_{Z}(\Omega)$.

$$
\kappa_{z}^{2}(\Omega)=\left(1-\frac{\Omega^{2}}{\gamma^{2}}\right)\left[\frac{\pi^{2}(m+1 / 4)^{2}}{\left(1-\Omega^{2}\right) \bar{b}^{2}(\delta)}-1\right] .
$$

The number $m$ in this equation is used in Sec. 4 to numerate the corresponding pairs of the dispersion curves with numbers $n=2 m+1$ and $n=2 m+2$ (see the lower inset in Fig. 5). In particular, the dispersion curve numbered by $m=0$ is actually the pair of curves with numbers $n=1$ and $n=2$ for antisymmetric and symmetric localized modes, respectively, that are close to each other.

Derived dispersion relations (27) and (34) are valid in relatively wide range of the frequencies and wave vectors, and in this range they reveal the anomalous dispersion of the localized modes (see Sec. 4 for details). Though the WKB approximation predicts the non-monotonicity of the dispersion curves, this feature is located near light line, $\Omega=\varepsilon^{1 / 2} \gamma \kappa_{Z}$, beyond the formal applicability of this approximation. Moreover, the WKB approximation does not capture the behavior of the dispersion curves in the frequency range close to $\omega_{J},|\Omega-1| \ll 1$. In the following 
subsection, we present the exact solution that is devoid of these drawbacks.

\subsection{Exact solution}

One can obtain exact solution of Eq. (15) in terms of the associated Legendre functions, see Appendix A. The symmetric and antisymmetric with respect to magnetic field solutions can be presented in the following forms:

$$
\begin{aligned}
& a(\xi)=a_{\text {sym }}\left[\frac{f_{p}\left(\xi_{0}-\xi\right)}{f_{p}\left(\xi_{0}\right)}-\frac{f_{q}\left(\xi_{0}-\xi\right)}{f_{q}\left(\xi_{0}\right)}\right], \\
& a(\xi)=a_{\text {asym }}\left[\frac{f_{p}\left(\xi_{0}-\xi\right)}{f_{p}^{\prime}\left(\xi_{0}\right)}-\frac{f_{q}\left(\xi_{0}-\xi\right)}{f_{q}^{\prime}\left(\xi_{0}\right)}\right],
\end{aligned}
$$

respectively. Here $a_{\text {sym }}$ and $a_{\mathrm{asym}}$ are integration constants, and

$$
f_{p}(\xi)=P_{v}^{\mu}[\tanh (\xi)], \quad f_{q}(\xi)=Q_{v}^{\mu}[\tanh (\xi)],
$$

where $P_{v}^{\mu}[\tau]$ and $Q_{v}^{\mu}[\tau]$ are associated Legendre functions of the first and second kind, respectively, with

$$
2 v+1=\sqrt{8\left(\Omega^{2}-1\right)^{-1} \kappa_{s}^{2}+1}, \quad \mu=i \kappa_{s} .
$$

The dispersion relations are defined by Eq. (20) taken at $\xi=+\delta$ with $a(\xi)$ substituted from Eqs. (36).

Now we show the transition of the exact solutions to the case of absence of the external dc magnetic field. When the dc field is close to zero, $h_{0} \rightarrow 0$, we can simplify Eqs. (36) in the following way. Firstly, we expand the argument of the associated Legendre functions,

$$
\tanh \left(\xi_{0}-\xi\right) \approx 1-\frac{1}{2} h_{0}^{2} \exp [2(\xi-\delta)]
$$

Then, the associated Legendre functions with argument $\tau$ close to 1 can be presented as linear combinations of functions $(1-\tau)^{ \pm \mu / 2}$, see Eq. (A14) in Apendix A. Therefore, taking into account that $\mu=i \kappa_{s}$, we get

$$
\begin{gathered}
f_{p}\left(\xi_{0}-\xi\right) \approx c_{1} \exp \left[-i \kappa_{s}(\xi-\delta)\right], \\
f_{q}\left(\xi_{0}-\xi\right) \approx c_{2} \exp \left[-i \kappa_{s}(\xi-\delta)\right]+c_{3} \exp \left[i \kappa_{s}(\xi-\delta)\right]
\end{gathered}
$$

where $c_{1}, c_{2}$ and $c_{3}$ are constants. Hence, the symmetric and antisymmetric solutions (36) obviously take the form

$$
a(\xi)=a_{\mathrm{sym}} \sin \kappa_{s} \xi, \quad a(\xi)=a_{\mathrm{asym}} \cos \kappa_{s} \xi,
$$

respectively, leading to the corresponding dispersion relations,

$$
\cot \kappa_{s} \delta=\frac{\varepsilon^{-1} \Omega^{2} \kappa_{s}}{\left(\Omega^{2}-1\right) \kappa_{d}}, \quad \tan \kappa_{s} \delta=-\frac{\varepsilon^{-1} \Omega^{2} \kappa_{s}}{\left(\Omega^{2}-1\right) \kappa_{d}},
$$

which are valid in the absence of external dc magnetic field (see Ref. 17 for details).

\section{Numerical analysis}

In this section we present the obtained analytical results in the graphic form and describe the effect of the dc magnetic field on the dispersion curves.

\subsection{The distribution of the dc and ac fields}

Figure 2 shows the spatial distributions of the normalized dc magnetic field $h_{d c}(\xi)$ (the red dashed curves) and the magnetic field $H_{y}(\xi)$ of the antisymmetric localized Josephson plasma modes (the solid blue curves) at $\Omega=0.98$ in the low frequency range (main panel) and $\Omega=1.12$ in the high frequency range (inset). The external dc magnetic field is uniform in the dielectric, $h_{d c}(\xi)=h_{0}=H_{0} / \mathcal{H}_{0}$, and penetrates into the layered superconductor in the form of the tails of the fictitious vortices, Eq. (11),

$$
h_{d c}(\xi)=\frac{1}{\cosh \left(\xi_{0}-\xi\right)}+\frac{1}{\cosh \left(\xi_{0}+\xi\right)} .
$$

The ac magnetic field $H_{y}^{s}(\xi)$ is defined by Eq. (18) with solutions (36) and is plotted in Fig. 2 in arbitrary units.

For the case of high frequencies, $\Omega>1$, the localized JPWs oscillate across the slab, while, for the low frequencies, $\Omega<1$, the electromagnetic fields oscillate near the interfaces and evanesce deep into the slab. In both cases, the dc field causes the change of the amplitude and wavelength of the ac field oscillations near the interfaces.

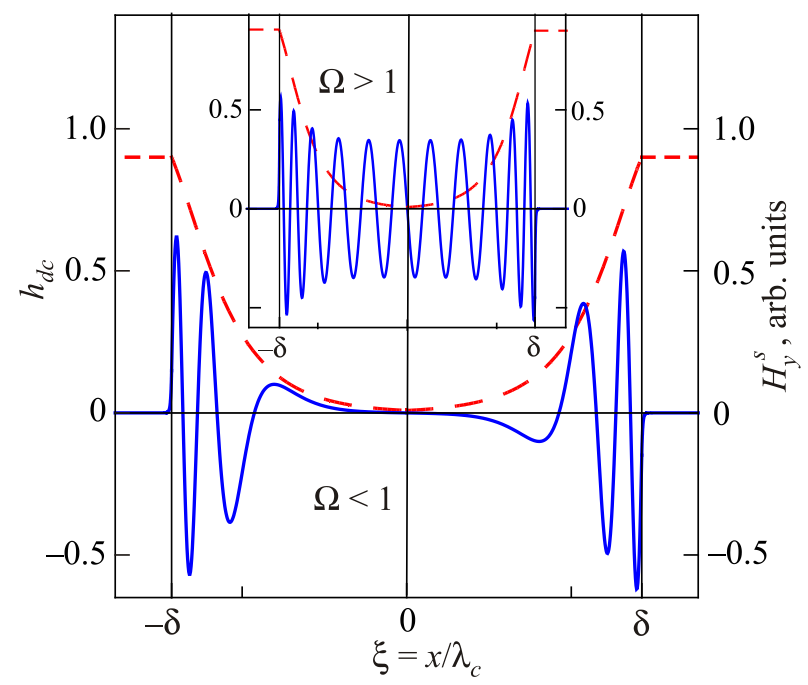

Fig. 2. (Color online) The spatial distribution of the ac magnetic field $H_{y}^{S}(\xi)$ (blue solid curves), in arb. units, and the normalized dc magnetic field (red dashed curves), $h_{d c}(\xi)$ ), for $\Omega=0.98$ (main panel) and $\Omega=1.12$ (inset). Parameters: $\gamma=5, \varepsilon=4, \kappa=10, \delta=5$, $h_{0}=0.9$. 


\subsection{Effect of the dc magnetic field and anomalous dispersion}

Firstly, we study the dispersion curves for relatively small $\kappa_{z} \lesssim 1$ in two frequency ranges, the low frequencies, $\Omega<1$, and the high frequencies, $\Omega>1$. For these cases, we plot the curves according to the exact solution obtained in Subsec. 3.2 and analyze their variation by changing the value $h_{0}$ of the external dc magnetic field. Secondary, we focus on the frequencies close to the Josephson plasma frequency $\omega_{J},|\Omega-1| \ll 1$, and the wider range over $\kappa_{z}$. Here, for certain value of $h_{0}$, we examine the transition from $\Omega>1$ to $\Omega<1$ in the behavior of the dispersion curves and compare the exact solution with the result obtained within the WKB approximation.

In order to simplify the following explanations, we numerate the dispersion curves from the bottom to top by $n=1,2,3, \ldots$ and study the shift of each curve by variation of the normalized amplitude $h_{0}$ of the external dc magnetic field.

We start the description from the low frequency range, $\Omega<1$. Figure 3 shows two lowest dispersion curves with numbers $n=1$ (thin solid lines for antisymmetric localized mode) and $n=2$ (thick dashed lines for symmetric localized mode) at $h_{0}=0, h_{0}=0.6, h_{0}=0.9, h_{0}=0.98$, and $h_{0}=0.999$. As seen, the dispersion curves shift towards the lower frequencies and increase their curvature when increasing the external dc magnetic field. The curves with $n=1$ and $n=2$ become close to each other with the increase of $h_{0}$.

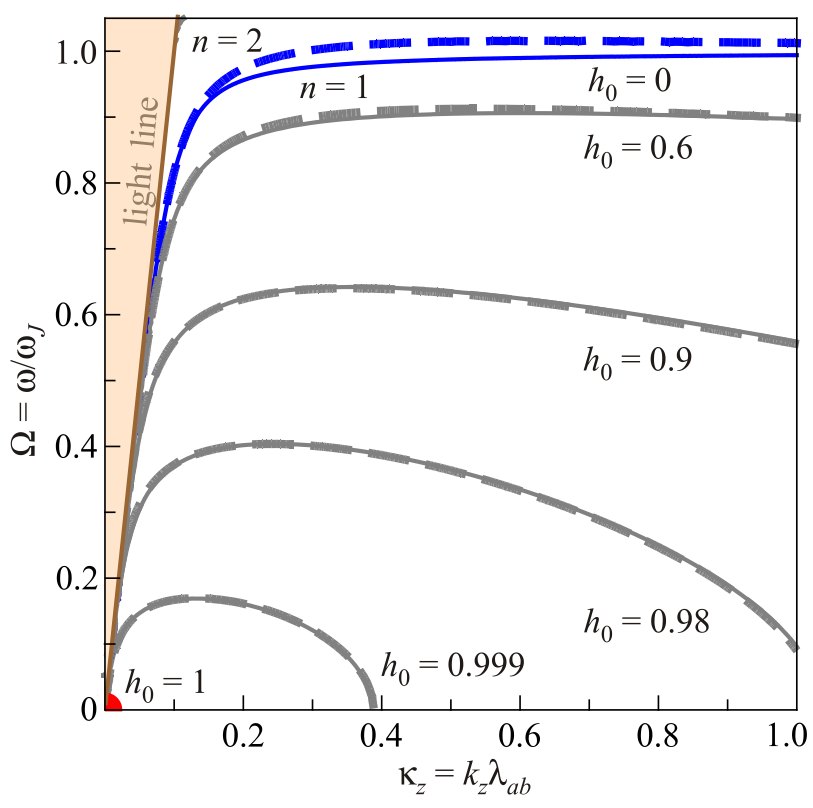

Fig. 3. (Color online) The dispersion curves with $n=1$ (thin solid lines, antisymmetric localized mode) and $n=2$ (thick dashed lines, symmetric localized mode) for $\Omega<1$ and the dc magnetic field $h_{0}$ : 0.999. The filled area above the light line, $\Omega=\varepsilon^{1 / 2} \gamma \kappa_{\mathrm{z}}$, presents the forbidden zone, where the localized modes do not exists. Other parameters: $\gamma=5, \delta=5, \varepsilon=4$.
This occurs because the symmetric and antisymmetric localized modes can be represented as two weakly coupled surface modes localized near interfaces $x=D / 2$ and $x=-D / 2$, and the coupling becomes weaker for the smaller values of $\Omega$. Moreover, when $h_{0} \rightarrow 1$, both curves can be described asymptotically by the same dispersion relation, $\kappa_{Z}^{2} / 3+\Omega^{2}\left[1+\left(\varepsilon \kappa_{d}\right)^{-1}\right]=\sqrt{1-h_{0}^{2}}$, and converge to the point $\kappa_{z}=0, \Omega=0$. Here $\kappa_{d}$ is defined by Eq. (21).

The dispersion curves presented in Fig. 3 (with the exception of the curve with $n=1$ at $h_{0}=0$ ), are non-monotonous and consist of the parts with normal (where $\partial \Omega / \partial \kappa_{z}>0$ ) and anomalous (where $\partial \Omega / \partial \kappa_{z}<0$ ) dispersions. Therefore, the curves have maximums, where the group velocity vanishes, $\partial \Omega / \partial \kappa_{z}=0$. These maximums appear near the light line, $\Omega=\varepsilon^{1 / 2} \gamma \kappa_{z}$ and shift when changing the amplitude of the external dc magnetic field. The possible application of this phenomenon is discussed in Subsec. 5.

Now we examine the high frequency range, $\Omega>1$. The corresponding dispersion curves are plotted in Fig. 4 by the solid ( $n=3,5, \ldots$ for antisymmetric localized modes) and dashed ( $n=4,6, \ldots$ for symmetric localized modes) lines for $h_{0}=0, h_{0}=0.6, h_{0}=0.9$, and $h_{0}=1$. Similarly to the case of low frequencies, the increase of the external dc magnetic field shifts the curves towards the lower frequencies (see the arrows in Fig. 4). The curves are nonmonotonous and have the parts with normal and anomalous dispersions. Comparing the curves for the intermediate fields $h_{0}=0.6$ and $h_{0}=0.9$ for different $n$, one can see that the shift due to the increase of $h_{0}$ is non-uniform and

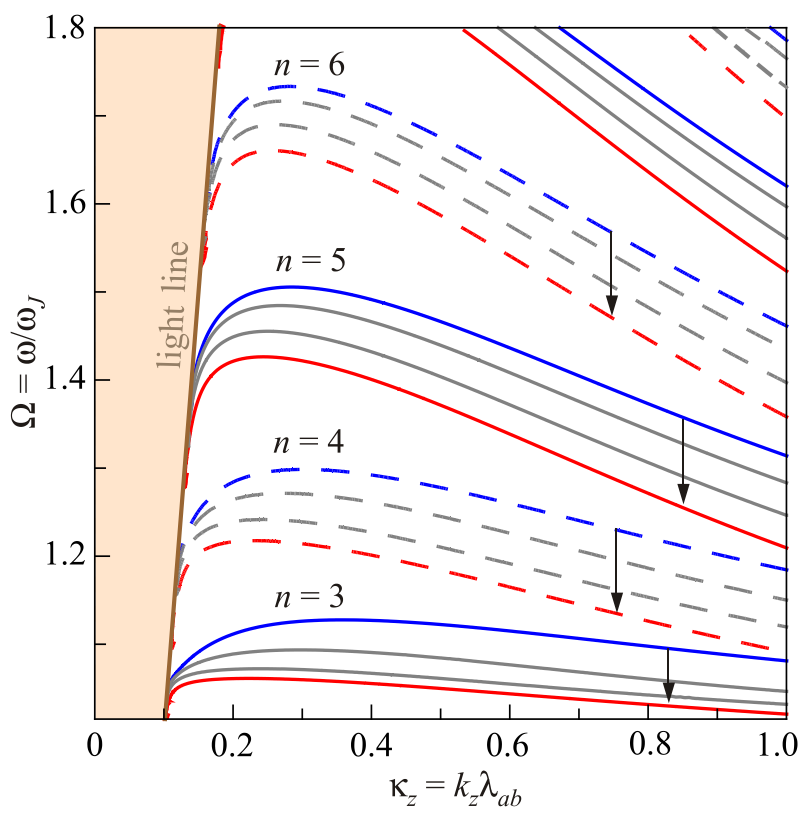

Fig. 4. (Color online) The dispersion curves for $\Omega>1$ and the dc magnetic field $h_{0}: 0,0.6,0.9$, and 1 plotted by solid $(n=3,5, \ldots$, antisymmetric localized modes) and dashed ( $n=4,6, \ldots$, symmetric localized modes) lines. The arrows show the increase of the field $h_{0}$. The other parameters and notations are the same as in Fig. 3. 
depends on $n$. When increasing $n$, the curves with $h_{0} \leq 0.6$ come close to each other, while the distance between curves with $h_{0} \geq 0.6$ increases. Figure 4 shows the curves for sufficiently small $\kappa_{z}$ and do not capture the interesting feature induced by the external dc magnetic field: at finite $h_{0}>0$ and sufficiently large $\kappa_{Z}$, all the dispersion curves with $n=3,4, \ldots$ intersect the line $\Omega=1$ and end at $\Omega=0$.

To study the mentioned feature, we proceed to the frequency range $|\Omega-1| \ll 1$ close to the Josephson plasma frequency. The main panel in Fig. 5 shows the behavior of two curves with $n=3$ and $n=4$ at $h_{0}=0.9$ in this frequency range for sufficiently large $\kappa_{z}$ while the upper inset in Fig. 5 shows the curves with $n=3,4,5,6$ in more narrow range of $\kappa_{z}$. The solid lines represent the exact dispersion curves, in accordance with Eqs. (20) and (36), while the dashed and dotted lines describe the dispersion curves obtained in the WKB approximation. The dashed curves are plotted using Eqs. (27) and (34), where we leave $\beta$ in the right-hand side. Though the WKB approximation formally is not valid close to the light line, $\Omega=\varepsilon^{1 / 2} \gamma \kappa_{z}$ where the maximums appear, one can see a good agreement between the solid and dashed curves for $\Omega$ not very close to 1 . The dotted curves are plotted using Eqs. (29) and (35), where we neglect $\beta$ in the right-hand side. The dotted curves are close to the solid ones for sufficiently great $\kappa_{z}$, where the WKB approximation is applicable, see condition (24).

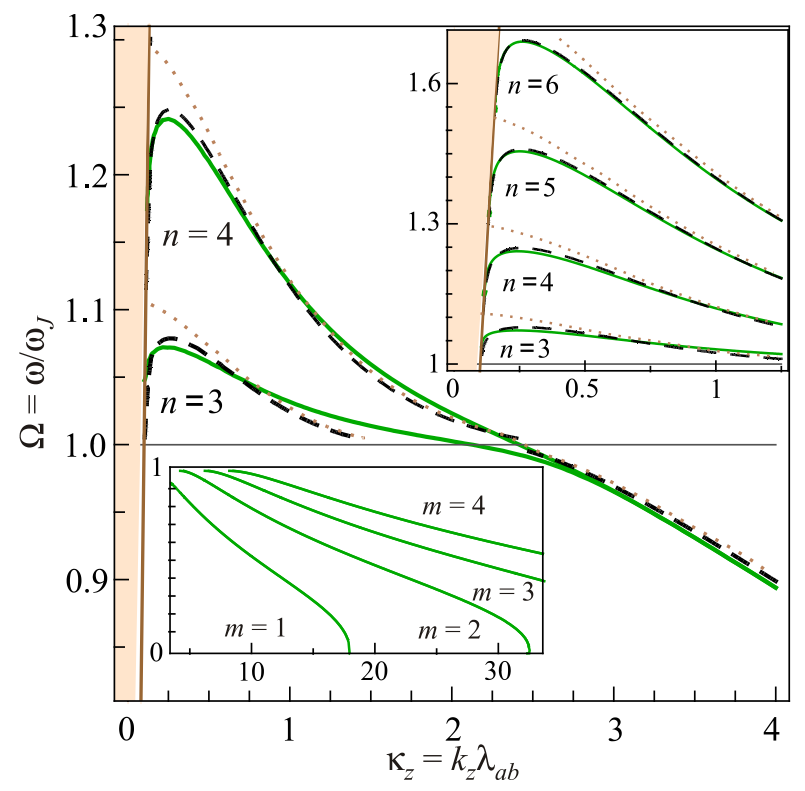

Fig. 5. (Color online) The dispersion curves with $n=3$, 4 (main panel) and $n=3$, 4, 5, 6 (upper inset) obtained for $h_{0}=0.9$. Solid lines are described by exact solution, Eqs. (20) and (36); dashed lines are plotted using the WKB formulas (27) and (34); dotted lines correspond to the simplified expressions Eqs. (29) and (35). The lower inset shows the pairs (numbered by $m=1,2,3,4$ ) of the dispersion curves with numbers $n=2 m+1$ and $n=2 m+2$, which are close to each other. The other parameters and notations are the same as in Fig. 3.
One can see from Fig. 5 that the curves with $n=3$ and $n=4$ intersect the line $\Omega=1$. Moreover, these curves come close to each other, when $\Omega<1$, due to the weak coupling between two interfaces. The same behavior is revealed by each pair of the dispersion curves, antisymmetric mode with $n=2 m+1$ and symmetric mode with $n=2 m+2$, with the same $m=1,2,3, \ldots$ The curves with $m=1,2,3,4$ are plotted in the lower inset of Fig. 5 for $\Omega<1$. In this frequency range, all the curves end at $\Omega=0$.

\section{Internal reflection of the localized modes in the inhomogeneous dc magnetic field}

In this section, we predict the internal reflection phenomenon that is related to the non-monotonous dispersion affected by the external dc magnetic field.

The dispersion curves discussed in the previous section are non-monotonous as functions $\kappa_{z}$ for fixed value of $h_{0}$. Therefore, for fixed value of $\Omega$, there are two values of $\kappa_{z}$ on each dispersion curve which correspond to the parts with the normal and anomalous dispersions. This means that the dispersion curves presented as functions $\kappa_{z}\left(h_{0}\right)$ for fixed value of $\Omega$ should be two-valued. This feature is shown in Fig. 6, where the dispersion curves with numbers $n=3$ (dash-dotted line), $n=4$ (dashed line), and $n=5$ (solid line) are plotted as functions $\kappa_{z}\left(h_{0}\right)$ for fixed values $\Omega=1.07, \Omega=1.25$, and $\Omega=1.5$, respectively.

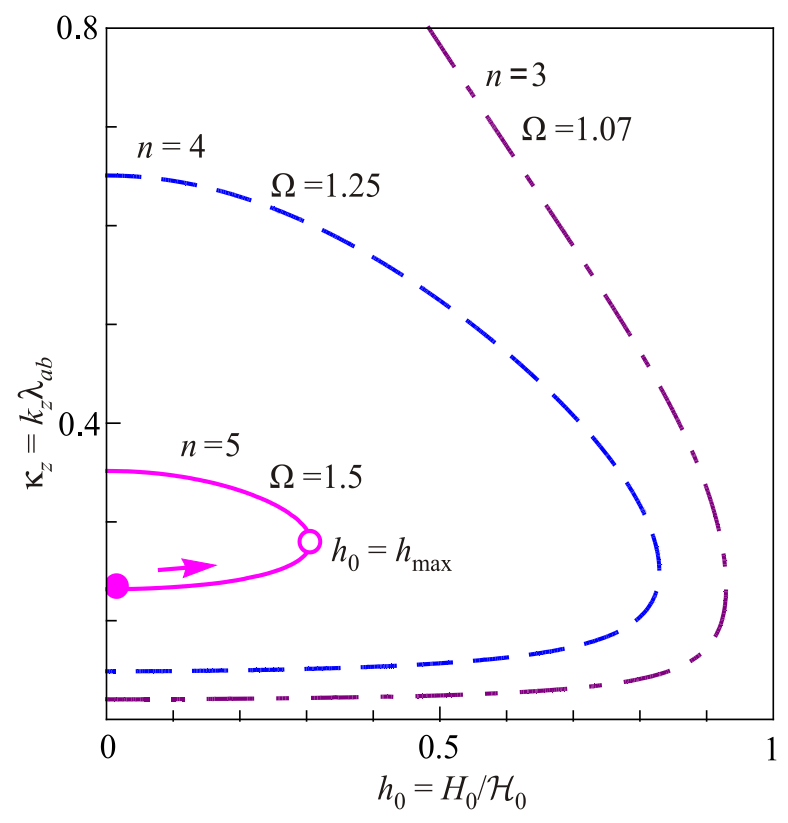

Fig. 6. (Color online) Dispersion curves with numbers $n=3$ (dash-dotted line), $n=4$ (dashed line), and $n=5$ (solid line) as functions $\kappa_{z}\left(h_{0}\right)$ for the fixed frequencies $\Omega=1.07, \Omega=1.25$, and $\Omega=1.5$, respectively. The arrow shows the variation of $\kappa_{z}$ along the mode propagation in the non-homogeneous dc magnetic field, while the solid and empty circles correspond to the initial ( $h=0$, $\left.\kappa_{z}=0.23\right)$ and critical $\left(h_{0}=h_{\max }=0.31, \kappa_{z}=0.28\right)$ points of this variation. Other parameters are the same as in Fig. 3. 
Now we presume that the localized mode propagates along the slab of the layered superconductor and that the external dc magnetic field is non-homogeneous and smoothly increases along the $z$-axis from $h_{0}=0$ to $h_{0}=0.5$. Considering, for example, the mode frequency $\Omega=1.5$ and the initial $z$-projection of the wave vector $\kappa_{z}=0.23$, we examine the solid curve in Fig. 6. When the external dc magnetic field increases along the propagation, $\kappa_{z}$ traces the curve (along the arrow in Fig. 6) from the initial point (marked as a solid circle) to the critical point (marked as an empty circle) with $h_{0}=h_{\max }=0.31$ and $\kappa_{z}=0.28$. After the critical point, when $h_{0}$ continues to increase, the wave vector should become imaginary, so the wave attenuates along the $z$-axis. Thus, in this point the localized mode should reflect, i.e., the phenomenon similar to the total internal reflection occurs. This phenomenon is of particular interest and can be used to control the localized mode propagation.

\section{Conclusions}

In this work, the effect of the external dc magnetic field on the Josephson plasma modes localized on a slab of layered superconductor is studied theoretically. The dispersion relations for the localized modes are obtained analytically within the WKB approximation and in the exact form in terms of the Legendre functions. The analysis is performed both for the frequencies $\omega$ higher and lower than the Josephson plasma frequency $\omega_{J}$. It is shown, that for the wide range of frequencies and wave numbers, the anomalous dispersion can be observed. The symmetry of the studied system implies the symmetry of the localized modes, symmetric and antisymmetric with respect to the ac magnetic field. For high and low frequency ranges, the localized modes have the different behavior. For $\omega>\omega_{J}$, the external dc magnetic field shifts relatively slightly the dispersion curves towards the lower frequencies. For $\omega<\omega_{J}$, the shift caused by the dc field is more significant. In this range, the localized modes evanesce deep into the slab, and waves near two interfaces are coupled weakly. Therefore, the dispersion curves for the symmetric and antisymmetric modes nearly coincide. Additionally, due to the dc magnetic field, all the dispersion curves starting at $\omega>\omega_{J}$ descend to $\omega<\omega_{J}$ at sufficiently great values of the wave vectors and end at $\omega=0$. The variation of the dispersion curves, when changing the dc magnetic field, reveals the way to control of the localized modes. In particular, we discuss the internal reflection of the localized modes propagating in the external inhomogeneous dc magnetic field. Discussed phenomenon can be applied in terahertz electronics and photonics for manipulation by the localized Josephson plasma modes.

We dedicate this paper to the memory of outstanding physicist-theoretician Alexei Alexeevich Abrikosov on the occasion of his 90th birthday. Gone, but not forgotten.
We gratefully acknowledge partial support from the grant of State Fund For Fundamental Research of Ukraine (Project No. 76/33683)

\section{Appendix A: Associated Legendre functions}

Here we describe how the solutions (36) can be derived in terms of the Legendre functions.

Firstly, we consider Eq. (15) with $u(\xi)$ taken in the form

$$
u(\xi)=\frac{2\left(1-\Omega^{2}\right)^{-1}}{\cosh ^{2}(\xi)} .
$$

Introducing new variable $\tau=\tanh (\xi)$ we can rewrite Eq. (15) as

$$
\left(1-\tau^{2}\right) a^{\prime \prime}(\tau)-2 \tau a^{\prime}(\tau)+\left[v(v+1)-\frac{\mu^{2}}{1-\tau^{2}}\right] a(\tau)=0
$$

where

$$
v(v+1)=2\left(1-\Omega^{2}\right)^{-1} \kappa_{s}^{2}, \quad \mu^{2}=-\kappa_{s}^{2} .
$$

The solutions of Eq. (A2) are $P_{v}^{\mu}(\tau)$ and $Q_{v}^{\mu}(\tau)$, the associated Legendre functions (see, e.g., [29]) of the first and second kinds, respectively,

$$
a(\tau)=C_{1} P_{v}^{\mu}(\tau)+C_{2} Q_{v}^{\mu}(\tau)
$$

or, returning to variable $\xi$,

$$
a(\xi)=C_{1} P_{v}^{\mu}[\tanh (\xi)]+C_{2} Q_{v}^{\mu}[\tanh (\xi)] .
$$

It should be noted that associated Legendre functions can be represented in the following form:

$$
\begin{aligned}
P_{v}^{\mu}(\tau)= & \frac{1}{\Gamma(1-\mu)}\left[\frac{1+\tau}{1-\tau}\right]^{\mu / 2} F[-v, v+1 ; 1-\mu ;(1-\tau) / 2], \\
& Q_{v}^{\mu}(\tau)=\frac{\sqrt{\pi} \Gamma(v+\mu+1)}{2^{v+1} \Gamma(v+3 / 2)} \frac{\left(1-\tau^{2}\right)^{\mu / 2}}{\tau^{v+\mu+1}} \times \\
& \times F\left[\frac{v+\mu+1}{2}, \frac{v+\mu+2}{2} ; v+\frac{3}{2} ; \frac{1}{\tau^{2}}\right]
\end{aligned}
$$

where $\Gamma(z)$ is the Euler gamma-function,

$$
\Gamma(z)=\int_{0}^{\infty} x^{z-1} \mathrm{e}^{-x} d x,
$$

and $F[a, b ; c ; z]$ is the hypergeometric function that is a solution (regular at $z=0$ ) of Eulers hypergeometric differential equation,

$$
z(1-z) F^{\prime \prime}+[c-(a+b+1) z] F^{\prime}-a b F=0,
$$

where prime denotes derivative with respect to $z$. The hypergeometric function can be also defined by power series, 


$$
F(a, b ; c ; z)=\sum_{n=0}^{\infty} \frac{(a)_{n}(b)_{n}}{(c)_{n}} \frac{z^{n}}{n !},
$$

where $(q)_{n}$ is the rising Pochhammer symbol

$$
(q)_{n}= \begin{cases}1, & n=0, \\ q(q+1) \cdots(q+n-1), & n=1,2, \ldots .\end{cases}
$$

Now we use general solution (A5) to find the symmetric and antisymmetric solutions of Eq. (15) with $u(\xi)$ defined by Eq. (16). For this purpose, we consider this equations only for $0<\xi<\delta$ with one of conditions (22). Since we presume relatively thick slabs, $\exp \left(D / \lambda_{c}\right) \gg 1$, we can reduce $u(\xi)$ to

$$
u(\xi)=\frac{2\left(1-\Omega^{2}\right)^{-1}}{\cosh ^{2}\left(\xi_{0}-\xi\right)},
$$

because the second term in the square brackets in Eq. (16) is negligible. Therefore, the general solution of Eq. (15) can be presented in the same form as Eq. (A4) but with changing $\xi$ to $\xi-\xi_{0}$,

$$
a(\xi)=C_{1} P_{v}^{\mu}\left[\tanh \left(\xi-\xi_{0}\right)\right]+C_{2} Q_{v}^{\mu}\left[\tanh \left(\xi-\xi_{0}\right)\right] .
$$

Finally, we apply one of conditions (22) to the last equation and achieve the solutions presented in Eqs. (36).

It worth noticing that the associated Legendre functions display the following asymptotic behavior,

$$
\begin{gathered}
P_{v}^{\mu}[\tau] \approx \frac{2^{\mu / 2}}{\Gamma(1-\mu)}(1-\tau)^{-\mu / 2}, \quad 0<1-\tau \ll 1, \\
Q_{v}^{\mu}[\tau] \approx \frac{\pi 2^{\mu / 2} \cot (\pi \mu)}{2 \Gamma(1-\mu)}(1-\tau)^{-\mu / 2}+ \\
+\frac{\pi^{2} 2^{-\mu / 2} \csc (\pi \mu) \csc [\pi(\mu+v)]}{2 \Gamma(1+\mu) \Gamma(-\mu-v) \Gamma(1-\mu+v)}(1-\tau)^{\mu / 2} .
\end{gathered}
$$

These asymptotic expressions are used in the main text to examine the case of weak dc magnetic fields, see Eq. (40).

1. R. Kleiner, F. Steinmeyer, G. Kunkel, and P. Müller, Phys. Rev. Lett. 68, 2394 (1992).

2. R. Kleiner and P. Müller, Phys. Rev. B 49, 1327 (1994).

3. L. Ozyuzer, A. E. Koshelev, C. Kurter, N. Gopalsami, Q. Li, M. Tachiki, K. Kadowaki, T. Yamamoto, H. Minami, H. Yamaguchi, T. Tachiki, K.E. Gray, W.K. Kwok, and U. Welp, Science 318, 1291 (2007).

4. M. Tonouchi, Nature Photon. 1, 97 (2007).

5. A. Dienst, E. Casandruc, D. Fausti, L. Zhang, M. Eckstein, M. Hoffmann, V. Khanna, N. Dean, M. Gensch, S. Winnerl, W. Seidel, S. Pyon, T. Takayama, H. Takagi, and A. Cavalleri, Nature Mater. 12, 535 (2013).

6. S. Rajasekaran, E. Casandruc, Y. Laplace, D. Nicoletti, G.D. Gu, S.R. Clark, D. Jaksch, and A. Cavalleri, Nature Phys. 12, 1012 (2016).
7. Y. Laplace, A. Cavalleri, Adv. Phys: X 1, No. 3, 387 (2016).

8. S. Savel'ev, V. Yampol'skii, and F. Nori, Phys. Rev. Lett. 95, 187002 (2005).

9. V.A. Golick, D.V. Kadygrob, V.A. Yampol'skii, A.L. Rakhmanov, B.A. Ivanov, and F. Nori, Phys. Rev. Lett. 104, 187003 (2010).

10. A.L. Rakhmanov, V.A. Yampol'skii, J.A. Fan, F. Capasso, and F. Nori, Phys. Rev. B 81, 075101 (2010).

11. V.A. Yampol'skii, A.V. Kats, M.L. Nesterov, A.Yu. Nikitin, T.M. Slipchenko, S. Savel'ev, and F. Nori, Phys. Rev. B 76, 224504 (2007).

12. D.V. Kadygrob, N.M. Makarov, F. Perez-Rodriguez, T.M. Slipchenko, and V.A. Yampol'skii, New J. Phys. 15, 023040 (2013).

13. V.M. Agranovich and D.L. Mills, Surface Polaritons, V.M. Agranovich and D.L. Mills (eds.), NorthHolland, Amsterdam (1982).

14. H. Raether, Surface Plasmons, Springer-Verlag, New York (1988).

15. R. Petit, Electromagnetic Theory of Gratings, Springer, Berlin (1980).

16. V.A. Yampol'skii, S. Savel'ev, O.V. Usatenko, S.S. Mel'nik, F.V. Kusmartsev, A.A. Krokhin, and F. Nori, Phys. Rev. B 75, 014527 (2007).

17. S.S. Apostolov, V.I. Havrilenko, Z.A. Maizelis, and V.A. Yampol'skii, Fiz. Nizk. Temp. 43, 360 (2017) [Low Temp. Phys. 43, 296 (2017)].

18. V.G. Veselago, Usp. Phys. Nauk 92, 517 (1967).

19. J.B. Pendry, Phys. Rev. Lett. 85, 3966 (2000).

20. W.J. Padilla, D.N. Basov, and D.R. Smith, Mater. Today $\mathbf{9}$, 28 (2006).

21. A. Goswami, S. Aravindan, P.V. Rao, and M. Yoshino, Crit. Rev. Solid State Mater. Sci. 41, 367 (2016).

22. S.S. Apostolov, Z.A. Maizelis, N.M. Makarov, F. PérezRodríguez, T. N. Rokhmanova, and V.A. Yampol'skii, Phys. Rev. B 94, 024513 (2016).

23. T.N. Rokhmanova, S.S. Apostolov, Z.A. Maizelis, and V.A. Yampol'skii, Fiz. Nizk. Temp. 42, 1167 (2016) [Low Temp. Phys. 42, 916 (2016)].

24. S.I. Khankina, V.M. Yakovenko, and V.A. Yampol'skii, Fiz. Nizk. Temp. 38, 245 (2012) [Low Temp. Phys. 38, 193 (2012)].

25. S. Savel'ev, V.A. Yampol'skii, A.L. Rakhmanov, and F. Nori, Rep. Prog. Phys. 73, 026501 (2010).

26. Ch. Helm and L.N. Bulaevskii, Phys. Rev. B 66, 094514 (2002).

27. V.A. Yampol'skii, D.R. Gulevich, Sergey Savel'ev, and Franco Nori, Phys. Rev. B 78, 054502 (2008).

28. S. Savel'ev, A.L. Rakhmanov, V.A. Yampol'skii, and F. Nori, Nature Phys. 2, 521 (2006).

29. A. Ango, Mathematics for Electro- and Radioengineers, Science, Moscow (1967). 